\title{
The Amplitude and Timing of the BOLD Signal Reflects the Relationship between Local Field Potential Power at Different Frequencies
}

\author{
Cesare Magri, ${ }^{1,2 *}$ Ulrich Schridde, ${ }^{1 *}$ Yusuke Murayama, ${ }^{1}$ Stefano Panzeri, ${ }^{3,4}$ and Nikos K. Logothetis ${ }^{1,5}$ \\ ${ }^{1}$ Max Planck Institute for Biological Cybernetics, 72076 Tübingen, Germany, ${ }^{2}$ Bernstein Center for Computational Neuroscience Tübingen, Germany, \\ ${ }^{3}$ Italian Institute of Technology, Department of Robotics, Brain and Cognitive Sciences, 16163 Genova, Italy, ${ }^{4}$ Institute of Neuroscience and Psychology, \\ University of Glasgow, Glasgow G12 8QB, United Kingdom, and ${ }^{5}$ Imaging Science and Biomedical Engineering, University of Manchester, Manchester M13 \\ 9PT, United Kingdom
}

There is growing evidence that several components of the mass neural activity contributing to the local field potential (LFP) can be partly separated by decomposing the LFP into nonoverlapping frequency bands. Although the blood oxygen level-dependent (BOLD) signal has been found to correlate preferentially with specific frequency bands of the LFP, it is still unclear whether the BOLD signal relates to the activity expressed by each LFP band independently of the others or if, instead, it also reflects specific relationships among different bands. We investigated these issues by recording, simultaneously and with high spatiotemporal resolution, BOLD signal and LFP during spontaneous activity in early visual cortices of anesthetized monkeys (Macaca mulatta). We used information theory to characterize the statistical dependency between BOLD and LFP. We found that the alpha $(8-12 \mathrm{~Hz})$, beta $(18-30 \mathrm{~Hz})$, and gamma $(40-100 \mathrm{~Hz}) \mathrm{LFP}$ bands were informative about the BOLD signal. In agreement with previous studies, gamma was the most informative band. Both increases and decreases in BOLD signal reliably followed increases and decreases in gamma power. However, both alpha and beta power signals carried information about BOLD that was largely complementary to that carried by gamma power. In particular, the relationship between alpha and gamma power was reflected in the amplitude of the BOLD signal, while the relationship between beta and gamma bands was reflected in the latency of BOLD with respect to significant changes in gamma power. These results lay the basis for identifying contributions of different neural pathways to cortical processing using fMRI.

\section{Introduction}

In recent years, blood oxygen level-dependent (BOLD) functional magnetic resonance imaging (fMRI) (Ogawa et al., 1992) has rapidly become the leading research tool in cognitive neuroscience. BOLD fMRI has been used extensively to investigate functional localization of neural activity associated with cognitive tasks or sensory processing. The BOLD signal, however, is only indirectly related to neural activity via a complex interplay of several factors including cerebral blood flow, cerebral blood volume, and cerebral metabolic rate of oxygen (D'Esposito et al., 2003; Brown et al., 2007; Logothetis, 2008). Thus, great caution is

Received Aug. 2, 2011; revised 0ct. 21, 2011; accepted Nov. 25, 2011.

Author contributions: C.M., U.S., S.P., and N.K.L. designed research; C.M., U.S., and Y.M. performed research; C.M. and S.P. contributed unpublished reagents/analytic tools; C.M., U.S., and S.P. analyzed data; C.M., U.S., Y.M., S.P., and N.K.L. wrote the paper.

This research was supported by the Max Planck Society, by the Neural Computation project of the Italian Institute of Technology, by the Compagnia di San Paolo, and was part of the research program of the Bernstein Center for Computational Neuroscience, Tübingen, Germany, funded by the German Federal Ministry of Education and Research (FKZ: 01GQ1002). We thank M. Augath for help with experiments; A. Oeltermann for developing the laminar electrode and data acquisition hardware; and M. Lindig and D. Ipek for preparing and maintaining animal anesthesia.

${ }^{*}$ C.M. and U.S. contributed equally to the work.

Correspondence should be addressed to Cesare Magri, Max Planck Institute for Biological Cybernetics, 38 Spemanstrasse, 72076 Tübingen, Germany. E-mail: cesare.magri@tuebingen.mpg.de.

DOI:10.1523/JNEUROSCI.3985-11.2012

Copyright $\odot 2012$ the authors $\quad 0270-6474 / 12 / 321395-13 \$ 15.00 / 0$ needed when inferring changes in neural activity from the temporal dynamics of the BOLD signal (Logothetis, 2008).

There is growing evidence, however, that the BOLD signal strongly correlates with the local field potential (LFP) (Logothetis et al., 2001; Thomsen et al., 2004; Viswanathan and Freeman, 2007; Rauch et al., 2008). The LFP is a mass neural signal that captures a multitude of neural processes, such as synaptic potentials, afterpotentials of somatodendritic spikes, and voltage-gated membrane oscillations, and that reflects the input of a given cortical area as well as its local intracortical processing, including the activity of excitatory and inhibitory interneurons and the effect of neuromodulatory pathways (Logothetis, 2003, 2008).

Previous studies showed that the BOLD signal correlates preferentially with specific frequency bands of the LFP (Logothetis et al., 2001; Kayser et al., 2004; Niessing et al., 2005; Goense and Logothetis, 2008; Murayama et al., 2010; Schölvinck et al., 2010). Substantial evidence suggests that different frequency bands of the LFP correlate with distinct behavioral states (Lindsley and Wicke, 1974; Steriade and Hobson, 1976; Basar, 1980) and reflect to a large extent the activity of different neural processing pathways (Belitski et al., 2008). Characterizing the relationship between the activity expressed by each LFP band and the BOLD contrast may thus allow the identification of contributions of different neural pathways to cortical processing using fMRI. To achieve this aim, it is necessary to understand whether the BOLD 
signal relates to each LFP band independently of the others or if, instead, the BOLD signal also reflects specific relationships among different LFP bands (Kilner et al., 2005; Rosa et al., 2010; Scheeringa et al., 2011).

To answer these questions, we recorded simultaneous and colocalized LFP and BOLD signals with high spatial and temporal resolution in the visual cortex of anesthetized macaques in the absence of visual stimulation. Considering spontaneous activity allows investigating the relationship between BOLD signal and neural activity without the strong biases and confounds which might be induced by stimuli. We computed mutual information, a principled measure of all possible statistical dependencies between any two signals, to determine which LFP bands are informative about the BOLD signal. We then demonstrated that the relationship between alpha and gamma power is reflected in the amplitude of the BOLD signal, while the relationship between the power of the beta and gamma bands is reflected by the delay with which changes in BOLD signal occur following changes in gamma power.

\section{Materials and Methods}

We performed seven combined electrophysiological fMRI recording sessions in four male adult anesthetized monkeys (Macaca mulatta, 4.6$10.4 \mathrm{~kg}$ ). All procedures were performed with great care to ensure the well-being of the animals, were approved by the local authorities (Regierungspräsidium), and were in full compliance with the guidelines of the European Community (EUVD 86/609/EEC) for the care and use of laboratory animals. A detailed description of the surgical procedures and preparation of the monkeys before the recordings can be found previously (Logothetis et al., 1999; Murayama et al., 2010).

Anesthesia for combined physiological and $f M R I$ experiments. All experiments were conducted under general anesthesia. Animals were artificially ventilated $\sim 1.4 \mathrm{~L} / \mathrm{min}, 24$ strokes/min (Servo Ventilator $900 \mathrm{C}$; Siemens), anesthesia was maintained with remifentanil ( $3 \mu \mathrm{g} / \mathrm{kg} / \mathrm{h}$, i.v., GSK), and mivacurium chloride (3-6 mg/kg/h, i.v., GSK) was applied as muscle relaxant. Animals were continuously infused with lactated Ringer's solution $(2.5 \%$ glucose, $10 \mathrm{ml} / \mathrm{kg} / \mathrm{h}$, i.v.). If needed, intravascular volume was maintained by administering colloids (hydroxyethyl starch, $10-20 \mathrm{ml} / \mathrm{kg} / \mathrm{h}$, i.v.). Throughout an experiment, the physiological state of the monkey was monitored and body temperature $\left(38-39.5^{\circ} \mathrm{C}\right)$, endtidal $\mathrm{CO}_{2}(\sim 33 \mathrm{mmHg})$, and oxygen saturation $(>95 \%)$ were kept tightly within normal limits. Two drops of $1 \%$ cyclopentolate hydrochloride (Alcon Pharma) were administered to each eye to achieve mydriasis, and hard contact lenses (Wöhlk) with the appropriate dioptric power were used to bring the eyes to focus on the stimulus plane.

The main reason for collecting neural responses during anesthesia is that anesthetized preparations allow longer data acquisition times and to study neurovascular coupling without the strong effects of animal state, including effects of attention and arousal that introduce additional complication in the interpretation of signals. Additionally, it has been shown that using remifentanil (an ultra-fast-acting $\mu$-opioid receptor agonist) has no significant effect on the neurovascular and neural activity of brain areas that do not belong to the pain matrix (Lund et al., 1994; Logothetis et al., 1999, 2009; Sereno et al., 2002; Goense and Logothetis, 2008; Goense et al., 2008; Zappe et al., 2008a,b). In particular, visual cortex does not bind remifentanil (Jones et al., 1991).

Combined functional magnetic resonance imaging and electrophysiological recordings. All experiments were conducted in a vertical $4.7 \mathrm{~T}$ Bruker BioSpec $47 / 40 \mathrm{v}$ scanner with a $40 \mathrm{~cm}$ diameter bore (Bruker), running the Bruker ParaVision software. The scanner was equipped with an actively shielded 26 -cm-diameter gradient coil, which could attain gradient strengths up to $50 \mathrm{mT} / \mathrm{m}$ in rise times as short as $180 \mu$ s. The monkey was sitting in an upright position in a custom-made primate chair. Signals were acquired using either a $30-\mathrm{mm}$ - (two experiments) or an $85-\mathrm{mm}$ diameter transmit/receive radiofrequency surface coil, placed around the recording chamber over the occipital cortex to increase MR signal around the electrodes.
To guide electrode insertion sagittal and/or axial fast low-angle shot (FLASH) sequences were acquired (Haase et al., 1986). Slices were aligned with the recording chamber (repetition time $(\mathrm{TR})=2000 \mathrm{~ms}$, echo time $(\mathrm{TE})=10 \mathrm{~ms}$, bandwidth $=50 \mathrm{kHz}$, flip angle $=50-70^{\circ}$, field of view $(\mathrm{FOV})=96 \times 96 \mathrm{~mm}$, matrix size $=256 \times 256$, slice thickness $=$ $1 \mathrm{~mm})$.

To ensure a constant quality of both electrophysiological and fMRI signals, monkeys were intermittently presented with visual stimulation in between periods of spontaneous activity recording. Stimuli were presented binocularly, initially with a super video graphics array fiber-optic system (Silent Vision, SV-7021, AVOTEC), and later using an in-house custom-made magnetic resonance-compatible visual stimulator. In both cases, fiber scopes were aligned to the fovea for each of the subject's eyes by a fundus camera (RC250, Zeiss). The visual field of the scopes was $30 \times 23^{\circ}$ with a spatial resolution of $800 \times 600$ pixels and a frame rate of $60 \mathrm{~Hz}$. The stimulus consisted of a high-contrast (100\%) full-field rotating $\left(180^{\circ} \mathrm{s}\right)$ polar checkerboard, with the direction of the rotation being reversed every second to minimize adaptation. Stimulus-on periods of $48 \mathrm{~s}$ were interleaved with stimulus-off periods (blank black screen) of the same length. This sequence was repeated four times.

Spontaneous activity was recorded in runs of $4 \mathrm{~min}$, with the monkey sitting in complete darkness with its eyes open and no direct visual stimulation (Logothetis et al., 2009). High-spatiotemporal resolution, multishot (two segments) gradient echo-echoplanar imaging scans were run for the acquisition of spontaneous activity [TR $=250$ or $125 \mathrm{~ms}$ (two experiments), $\mathrm{TE}=20 \mathrm{~ms}$, bandwidth $=100 \mathrm{kHz}$, flip angle $=20-25^{\circ}$, yielding an intervolume time of 500 or $250 \mathrm{~ms}$, respectively. The FOV was $96 \times 96 \mathrm{~mm}$ or $96 \times 48 \mathrm{~mm}$ (two experiments) with a matrix size of $128 \times 128$ or $128 \times 64$ (two experiments) and a slice thickness of $2 \mathrm{~mm}$, resulting in a voxel resolution of $750 \times 750 \times 2000 \mu \mathrm{m}$. Dummy scans were run before all functional image acquisitions to ensure full saturation of the signal. After functional recordings, a FLASH sequence was run for anatomical reference $(\mathrm{TR} / \mathrm{TE}=2000 / 10 \mathrm{~ms}$, bandwidth $=50 \mathrm{kHz}$, flip angle $=70^{\circ}, \mathrm{FOV}=96 \times 96 \mathrm{~mm}$, matrix size $=256 \times 256$, slice thickness $=2 \mathrm{~mm}$ ). In two experiments, anatomical scans were acquired using GEFI sequences $(\mathrm{TR} / \mathrm{TE}=2000 / 10 \mathrm{~ms}$, bandwidth $=80 \mathrm{kHz}$, flip angle $=30^{\circ}, \mathrm{FOV}=96 \times 48 \mathrm{~mm}$, matrix size $=512 \times 256$, slice thickness $=0.5 \mathrm{~mm}$ ).

For electrophysiological recordings, a small skull trepanation was performed, the dura was left intact, and electrodes were slowly advanced into visual areas under visual and auditory guidance using custom-built microdrives. In two experiments, electrodes consisted of single glass-coated platinum-iridium wires $\left(\mathrm{Pt}_{90} \mathrm{Ir}_{10}\right.$; diameter $\left.18 \mu \mathrm{m}\right)$ (Logothetis et al., 2001). In all other experiments, multichannel electrodes were used (Murayama et al., 2010). These electrodes contained 10 single electrode leads of $18 \mu \mathrm{m} \mathrm{Pt} \mathrm{t}_{90} \mathrm{Ir}_{10}$ wire spaced by either 400 or $800 \mu \mathrm{m}$. The angle of the multichannel electrode penetration was such that electrode leads were located both in V1 and V2 (Fig. $1 A$ ). Due to the fact that four amplifiers were available in our combined electrophysiology-fMRI setup, we selected 4 of the 10 available electrode leads in the initial phase of the experiment. The selection was based on the following criteria. First, we assessed the anatomical location of the leads. This was done based solely on the magnetic resonance scans and by referring to a monkey brain atlas (Saleem and Logothetis, 2007). Subsequently, we tested in succession each one of the leads whose anatomical location was determined to be in $\mathrm{V} 1$ or in V2, and we selected the four leads that exhibited the strongest spiking/multiunit activity (MUA) response (defined as the power of the extracellular signal in the range $900-3000 \mathrm{~Hz}$ ). It is important to note that this selection was based only on the strength of the recorded response and not on any specific response pattern. Due to the angle of the electrode penetration and the chosen slice orientation and slice thickness of the magnetic resonance images, we could not unambiguously determine the laminar distribution of the recording contacts in our experiments. For the single glass-coated platinum-iridium electrodes, recording leads were referenced to the electrode holder, which consisted of a concentric metallic cylinder (either copper beryllium or a bronze alloy) (Logothetis et al., 2001; Oeltermann et al., 2007). In case of the multichannel electrodes, a flattened Ag wire, which was glued to the carbon shaft, served as reference (Murayama et al., 2010). In both cases, 
A

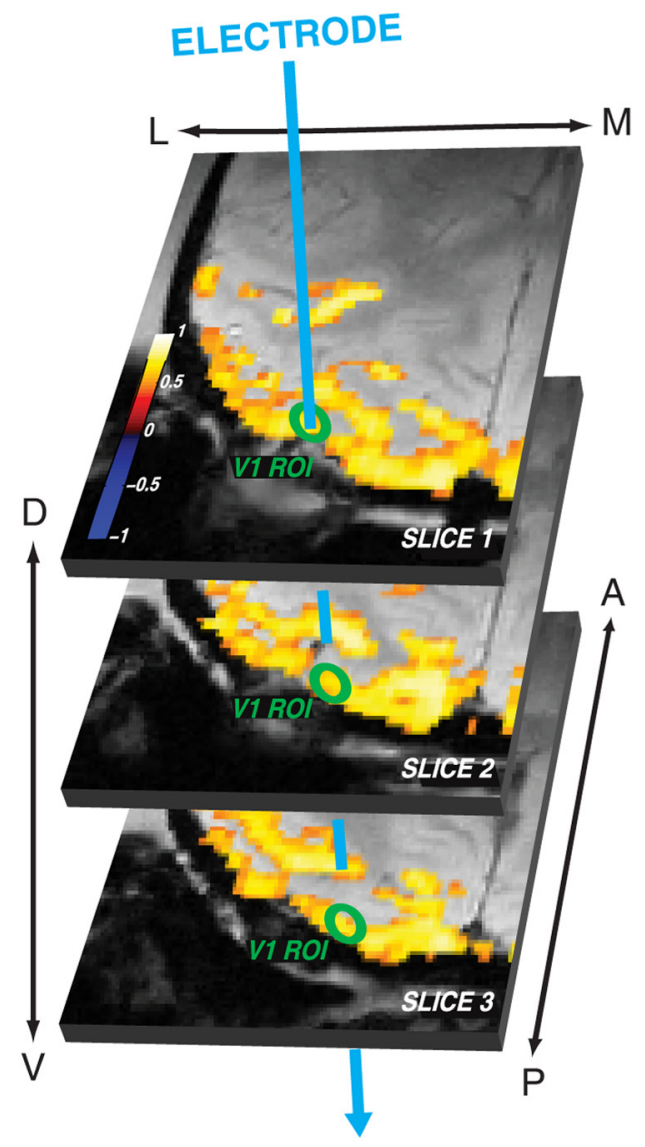

B
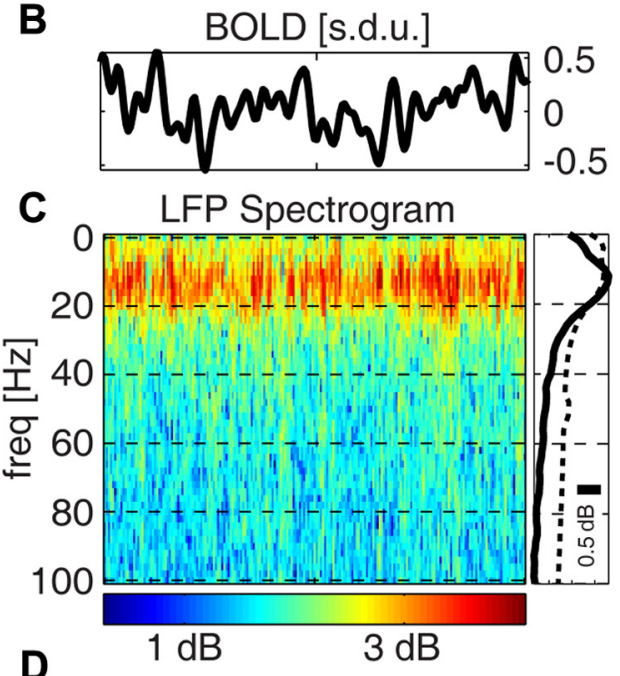

D
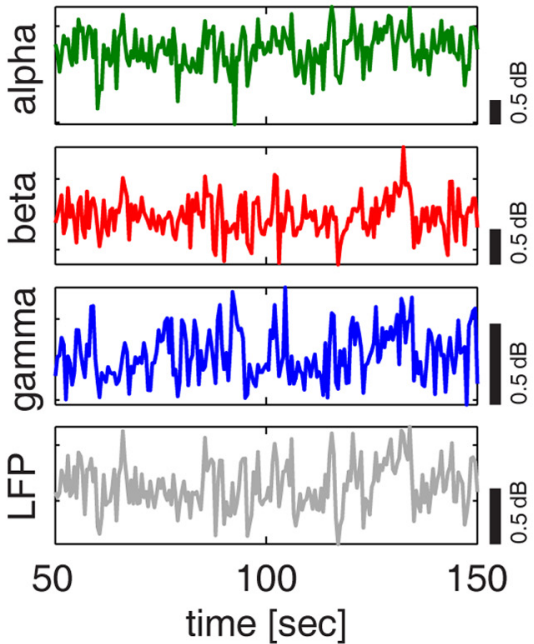

Figure 1. Example of combined electrophysiological and BOLD fMRI recording and illustration of the spectral properties of the LFP. $A$, Functional BOLD activation maps, superimposed on the anatomical scans, in response to a high-contrast (100\%), full-field, rotating polar checkerboard presented to both eyes. The activation maps are shown as the correlation coefficients $(p<0.0001$, $t$ test, uncorrected for multiple comparisons) between the BOLD signal and a boxcar model of the stimulus sequence convolved with a canonical hemodynamic response function. The green circles indicate a schematic ROI located in V1. The blue arrow indicates, for each slice, the approximate position of the multichannel electrode. $\boldsymbol{B}$, Example BOLD signal time course, averaged over an ROI located in V1, during spontaneous activity. Only $100 \mathrm{~s}$ are shown. C, Spectrogram of the LFPs recorded in V1 simultaneously with the BOLD signal shown in $\boldsymbol{B}$. The average spectrum over the plotted period is shown on the right as a solid black line. The dashed line illustrates the average spectrum computed using data from all sessions. $\boldsymbol{D}$, Time course of the spontaneous $L F P$ power in the alpha, beta, and gamma bands (green, red, and blue lines, respectively) and total LFP power (gray line).

reference contacts rested in the recording chamber, which was filled with a mixture of $0.9 \% \mathrm{NaCl}$ dissolved in deuterium water and $0.6 \%$ agaragar, $\mathrm{pH}$ 7.4, solution, which guaranteed good electrical connections between the ground contacts and the animal (Oeltermann et al., 2007). The electrodes had impedances between 327 and $600 \mathrm{k} \Omega$. Signals were recorded broadband, amplified, and digitized at $20.83 \mathrm{kHz}$ using a 16 bit analog-to-digital converter (PCI-6052E; National Instruments). All procedures used for recordings and on-line hardware-driven cleaning of the electrophysiological signals as well as the specifications of the recording hardware and electrodes have been described in detail previously (Logothetis et al., 2001; Oeltermann et al., 2007; Murayama et al., 2010).

Interpretation of LFP measures rests on well established biophysical findings and models and depends upon the size, geometry, and laminar structure of the neuronal generators (Nicholson, 1973; Mitzdorf, 1985; Schroeder et al., 1995, 1998; Logothetis, 2003; Pettersen et al., 2006). Considering our recording configurations and the well established directional specificity of the LFP in laminated cortical structures, we assume that a prominent contribution to the recorded LFPs comes from excitatory and inhibitory synaptic potentials, mostly from pyramidal neurons (Klee et al., 1965; Creutzfeldt et al., 1966a,b), but perhaps also from stellate cells (Murakami and Okada, 2006) and from afterdischarges not directly related to cellular activity (Granit et al., 1963; Mitzdorf, 1987; Kamondi et al., 1998; Juergens et al., 1999).

Data processing. All data were analyzed off-line, using custom-written software developed in Matlab (MathWorks). fMRI data were detrended and spatially filtered (Gaussian kernel, $3 \times 3$ pixels with $1.5 \sigma$ ), and the signal in each voxel was converted into SD units. The BOLD signal was then averaged over regions of interest (ROIs) centered around the electrode that were defined in V1 and in V2 using the anatomical scans as reference and referring to a monkey brain atlas (Saleem and Logothetis, 2007). The number of voxels in each ROI ranged from 90 to 130 for V1 and from 70 to 100 for V2. Because respiration was kept constant in all experiments to 24 strokes per min (corresponding to $0.417 \mathrm{~Hz}$ ), the BOLD signal was low-pass filtered with a cutoff of $0.3 \mathrm{~Hz}$ to remove respiratory artifacts.

The electrophysiological recordings were decimated to $7 \mathrm{KHz}$. A denoising procedure was applied to remove artifacts caused by the changes in the gradient changes of the electromagnetic fields (Logothetis et al., 2001; Murayama et al., 2010), and the $50 \mathrm{~Hz}$ line interference was removed.

Spectral estimation and band-limited power. LFP spectra were computed using 0.5 -s-long nonoverlapping windows. The window length 
was chosen to be $0.5 \mathrm{~s}$ to match the intervolume time of the fMRI acquisitions and corresponded to 3500 points of the electrophysiology time series sampled at $7 \mathrm{KHz}$. For each time window, the spectrum was obtained using the multitaper technique (Thomson, 1982; Percival and Walden, 1993), which provides an efficient way to simultaneously control the bias and variance of spectral estimates by using multiple Slepian data tapers. We used three data tapers (corresponding to a bandwidth parameter $N W=2$ ), which provide a good tradeoff between the variance in the spectral estimates and the frequency resolution of the spectra. The LFP spectrogram in Figure $1 C$ was generated using this procedure. The population average spectrum of Figure $1 C$ was obtained by taking the mean of the spectra over all time windows and by averaging over all recordings. We also quantified the total power within specific frequency ranges (e.g., the alpha band $8-12 \mathrm{~Hz}$ ). This band-limited power was computed by integrating the power in the spectra within the selected frequency band.

Mutual information. The aim of our information-theoretic analysis was to quantify the relationships between a neurophysiological signal (either the power of the LFP spectra at a single frequency value or the power of LFPs in a given frequency band, or MUA, defined as the power of the extracellular signal in the band between 900 and $3000 \mathrm{~Hz}$ ) and the BOLD signal. Mutual information [introduced by Shannon (1948) and abbreviated to "information" in the following] is the most general measure of the statistical dependency between any two signals because it depends on the full probabilities of observing the two considered signals jointly and thus takes into account all possible ways in which the two signals carry information about each other. As a result, information can capture the contributions of correlations at all orders. Moreover, and unlike for example the general linear model (Friston et al., 1994; Worsley and Friston, 1995), it can also properly capture the effect of any nonlinearity between the two signals (Fuhrmann Alpert et al., 2007). Having said this, it is trivial to model nonlinear dependencies within the general linear model by including nonlinear terms (e.g., polynomial expansions or classical interactions). It is important to note, however, that information is a symmetric measure that does not quantify causal dependencies between variables. Information values should thus only be interpreted as measures of statistical dependency.

A caveat of the present information analysis is that, although it can capture all types of nonlinear interactions between BOLD and LFP power, it does not investigate the phase relationship between BOLD and LFP. Therefore, the present study cannot rule out the presence of dependencies of BOLD upon LFP phase or upon nesting of rhythms.

To compute information, we proceeded as follows. We quantized both the BOLD and the neural signal into five levels, so that each level contained $20 \%$ of the recorded values. We denote by $B$ and $P$ the set of possible values taken by the quantized BOLD and by the quantized neural signals, respectively. We then computed the mutual information (Shannon, 1948) as follows:

$$
I(B ; P)=\sum_{p} P(p) \sum_{b} P(b \mid p) \log \frac{P(b \mid p)}{P(b)},
$$

where $P(b)$ and $P(p)$ are the probability of the BOLD signal and of the neural signal of taking a given value $b$ and $p$, respectively. $P(b \mid p)$ is the probability of observing a given value $b$ in the BOLD signal when the neural signal takes the value $p$. Since the experimental evaluation of these probabilities is made from a limited number of experimental observations, the resulting information measures suffer from a limited sampling bias (Panzeri et al., 2007). We corrected for this bias using the correction developed by Panzeri and Treves (1996), which is computationally fast and precise in the data-sampling regime considered here (Panzeri et al., 2007). We then checked for residual bias by a bootstrap procedure: the data points from the neuronal and the BOLD signal were paired at random, and the information for these random pairings was computed. Because in this random case the information should be zero, the resulting value is an indication of a residual error. The bootstrap estimate of this error was therefore removed. Additionally, we used the distribution of bootstrap values as the null hypothesis distribution for testing the significance of positive information values.
Complementary information. The above single-frequency information analysis can be extended to compute the amount of information about the BOLD signal that is obtained when jointly observing the power $p 1$ and $p 2$ in two different LFP frequency bands. The mutual information that the joint knowledge of the powers $p 1$ and $p 2$ conveys about the BOLD signal is defined as follows:

$$
I(B ; P 1 P 2)=\sum_{p 1 p 2} P(p 1 p 2) \sum_{B O L D} P(b \mid p 1 p 2) \log _{2} \frac{P(b \mid p 1 p 2)}{P(b)},
$$

where $P(b \mid p 1 p 2)$ is the probability of observing a given value $b$ in the BOLD signal when the LFP power takes the value $p 1$ in the first band and $p 2$ in the second band and $P(p 1 p 2)$ is the overall probability of observing powers $p 1$ and $p 2$.

We say that the information conveyed by $P 2$ is complementary to that conveyed by $P 1$ when the information $I(B ; P 1 P 2)$ carried by the joint observation of the power of both bands is higher than the information $I(B ; P 1)$ carried by $P 1$ only. This means that some information about the BOLD signal that is not available in the power of one band is available in the power of the other band. We quantify the amount of complementary information about the BOLD signal provided by signal $P 2$ over that provided by signal $P 1$ as follows:

$$
G A I N=I(B ; P 1 P 2)-I(B ; P 1) .
$$

The amount of complementary information can be expressed as the percentage information increase as follows:

$$
\% G A I N=\frac{I(B ; P 1 P 2)-I(B ; P 1)}{I(B ; P 1)} .
$$

The \%GAIN quantifies the amount of complementary information added by a second signal as the percentage of the information already present in the first signal. Note that the information gain simply reports the extra information or predictability on observing a second variable that cannot be explained by the first. However, this does not mean that the predictions of the first variable depend upon the second and thus cannot be interpreted as three-way mutual information.

The information $I(B ; P 1 P 2)$ was computed with the same discretization and bias subtraction procedure described above for $I(B ; P)$. However, we performed an additional test on the complementarity values. For each of the five classes in which we discretized the neural signal $P 1$, we randomly permuted the order of the signal $P 2$ within the class of $P 1$. This shuffling destroys only the additional information carried by the second power and hence provides a way to obtain a null hypothesis distribution of information values under the assumption that the power of the second band does not add any information beyond that already provided by the power of the first band. We repeated the random permutations 20 times, each time computing the resulting joint information $I(B ; P 1 P 2)$, and we used these values to construct the "null hypothesis distribution" that $P 2$ does not add information about $B$ beyond that of $P 1$.

\section{Results}

We performed simultaneous recordings of spontaneous neural activity and BOLD fMRI in visual areas V1 and V2 of four anesthetized monkeys. Electrophysiological signals were recorded from 9 sites in V1 from all monkeys and from 14 sites in V2 from two monkeys, resulting in a total of 23 recording sites. Approximately $2 \mathrm{~h}$ of spontaneous activity were recorded from each site. To investigate the relationship between the BOLD signal and the electrophysiology at the same location, we evaluated the BOLD signal in ROIs at the sites of the electrophysiological recording in $\mathrm{V} 1$ and V2 (Fig. $1 A$ ). A representative example (session I02tv1) of BOLD time series recorded in V1 is shown in Figure $1 B$. The spectrum of the neural activity (Fig. $1 C$ ) showed a broadband spectrum with sustained power over the whole range $0-100 \mathrm{~Hz}$ and with the power monotonically decreasing after an initial peak 
A I02tv1 - V1 site
Single Frequency Information [bits]
B 102tv1

Band Information
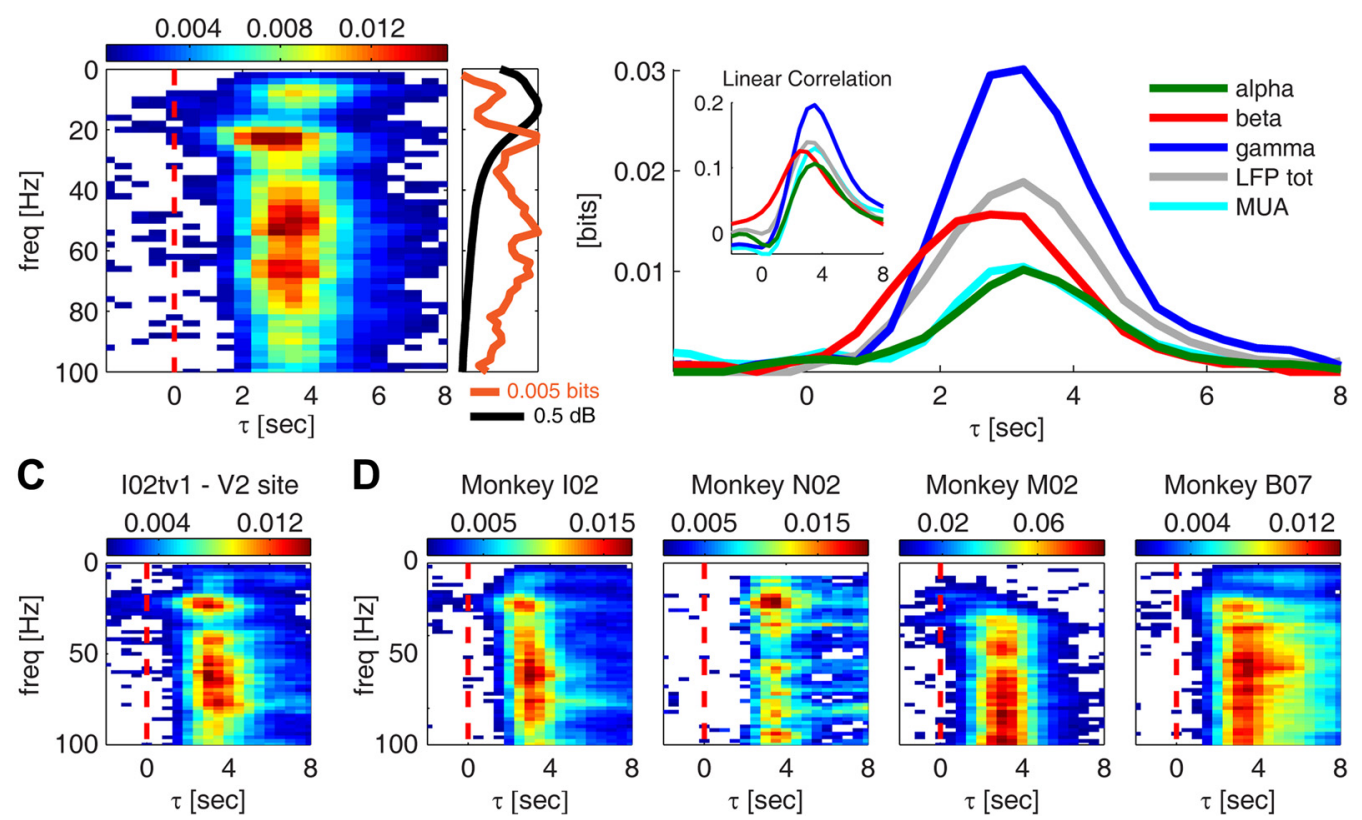

Figure 2. Information about the BOLD signal conveyed by LFP power at different frequencies. $A$, Mutual information between the BOLD signal and LFP power at different frequencies for different lags $\tau$ between the two signals ( $\tau>0$ indicates that the BOLD signal has been shifted back with respect to the neural response). Results are shown for a representative session (I02tv1). Only significant ( $p<0.05$, bootstrap test, uncorrected for multiple comparisons) information values are colored. On the right, the information values are plotted only for lag $\tau=3 \mathrm{~s}$ (orange line). The average spectrum of the LFPs recorded during the session is also shown (black line). $\boldsymbol{B}$, Mutual information between the BOLD signal and the LFP power in selected frequency bands and between the BOLD signal and MUA (same session as in $A$ ). The following four LFP bands are considered: alpha ([8-12 Hz], green line); beta ([18 - 30 Hz], red line); gamma ([40 - $100 \mathrm{~Hz}]$, blue line); the total LFP power ( $[0-100 \mathrm{~Hz}]$, gray line); and MUA is computed as the power of the electrophysiological signal between 900 and $3000 \mathrm{~Hz}$ (cyan line). The inset shows the Pearson correlation between the BOLD signal and the power in the LFP bands, and between the BOLD signal and MUA. C, Same plot as in $\boldsymbol{A}$ for a V2 recording site of session 102tv1. D, Same plot as in $\boldsymbol{A}$ for four additional sessions. One additional session is shown for monkey 102 , while one representative session is shown for each of the other monkeys.

at $\sim 10 \mathrm{~Hz}$. In the following, we systematically investigate the relationship between this broadband range of frequencies and the BOLD signal.

\section{Three LFP bands carry information about the BOLD signal}

To avoid committing too early to assumptions about a specific partitioning of the LFP into bands, we began our analysis by considering the relationship between the BOLD signal and the LFP power at single frequencies. We computed the mutual information between each LFP frequency and the BOLD signal for different values of the delay $\tau$ introduced between the neural and the BOLD signal. The parameter $\tau$ was varied between -2 and $10 \mathrm{~s}$, with positive values of $\tau$ indicating that the BOLD signal has been shifted back with respect to the neural response or, equivalently, that the LFP recorded at time 0 is aligned to the BOLD signal recorded at time $\tau$.

The result of this analysis is shown in Figure $2 \mathrm{~A}$ for a representative session (I02tv1). We found significant mutual information between the neural activity and the BOLD signal only for $\tau>$ 0 , as expected, due to the fact that in early visual cortices changes in neural activity precede those in the BOLD signal. Additionally, we observed three distinct LFP frequency regions that carried high information about BOLD: the $8-12,18-30$, and $40-100 \mathrm{~Hz}$ ranges. In the following, we will refer to these three frequency ranges as to the alpha, beta, and gamma bands, respectively, because their frequency ranges approximately correspond to rhythms described and termed this way in the EEG literature. However, it is important to note that with this partition and naming of the LFP frequency range we do not imply the existence of well separated rhythms corresponding to ambient EEG rhythms documented in the waking animal. These three frequency ranges are, in fact, not clearly separable as distinct peaks in the LFP spectrum (Figs. $1 C, 2 A, 3 A$ ). We decided to separate them based on how they carry information about BOLD, because we were able to recognize these three informative frequency regions across all monkeys (Fig. $2 \mathrm{D}$ ) and in both $\mathrm{V} 1$ and $\mathrm{V} 2$ recordings (Fig. 2C), and because (as we shall report in the following subsections) these three frequency regions had well identifiable and largely distinct relationships with the BOLD signal. The complementary relationship with the BOLD signal of these frequency ranges partly supports the interpretation of these bands as reflecting partly different putative sources of neural activity, although most likely these putative sources may not be completely separable by spectral analysis.

To understand whether neural activity integrated over a frequency range was a better predictor of the BOLD signal than the power of a single-frequency bin, we computed the information about BOLD conveyed by the total power in each of the three informative bands individuated above. For the example session (I02tv1), we found (Fig. 2B) that gamma was by far the most informative band ( 0.03 bits) followed by beta $(0.015$ bits $)$ and alpha $(0.01$ bits $)$. We also found that the information conveyed by the total $(0-100 \mathrm{~Hz})$ LFP power was much lower $(0.02$ bits $)$ than the information of gamma power and was comparable to that of beta power. The differences in information between these frequency bands suggest that the BOLD signal relates preferentially to specific LFP frequency regions.

Moreover, there was a clear difference in the temporal profile of the information carried by each band. Beta information increased and peaked earlier than alpha and gamma information, 
A Population
Single Frequency Information [bits] B

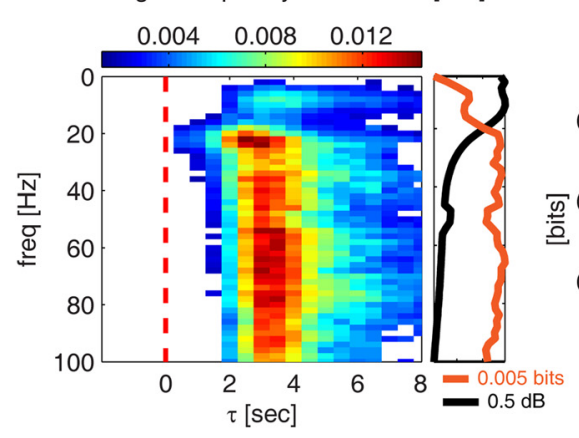

Population

Band Information

Figure 3. Session summary of the information results. All results are shown as median over all sessions. $A$, Mutual information between BOLD signal and LFP power at different frequencies for different lags $\tau$ between the two signals ( $\tau>0$ indicates that the BOLD signal has been shifted back with respect to the neural response). 0 nly significant ( $p<0.0001$, bootstrap test, uncorrected for multiple comparisons) information values are colored. On the right, the information values are plotted only for lag $\tau=3 \mathrm{~s}$ (orange line). The average spectrum of the LFPs is also shown (black line). $\boldsymbol{B}$, Mutual information between the BOLD signal and the LFP power in selected frequency bands, and between the BOLD signal and MUA. The following four LFP bands are considered: alpha ([8-12 Hz], green line); beta ([18 - 30 Hz], red line); gamma ([40-100 Hz], blue line); and the total LFP power ([0 - $100 \mathrm{~Hz}]$, gray line); and MUA is computed as the power of the electrophysiological signal between 900 and $3000 \mathrm{~Hz}$ (cyan line). The inset shows the Pearson correlation between the BOLD signal and different LFP bands, and between the BOLD signal and MUA. Solid lines indicate the median over all sessions, and shaded areas indicate the range between the 40th and 60th percentile.

suggesting that changes in the activity of this band are reflected earlier by changes occurring in the BOLD signal. The difference in $\tau$ between the peak of the information conveyed by beta power and that conveyed by alpha or gamma power was $0.5 \mathrm{~s}$.

These results were confirmed at the population level (Fig. 3B). Since we did not find any appreciable difference in the information profile of V1 and V2 recordings, here and in the following, we pooled V1 and V2 cases together to compute the population median. Gamma power conveyed the largest amount of information (median, 0.034 bits; 40th to 60th percentile, 0.029-0.035 bits; uncorrected for multiple comparisons), followed by beta (median, 0.017 bits; 40th to 60th percentile range, 0.015-0.019 bits; uncorrected) and alpha (median, 0.007 bits; 40th to 60th percentile range, $0.005-0.008$ bits; uncorrected). The information conveyed by the total LFP power (median, 0.02 bits; 40 th to 60th percentile range, $0.017-0.024$ bits; uncorrected) was only slightly higher than that of beta power. The information values were significant for all bands ( $p<0.05$; bootstrap test; uncorrected). The peak of the information conveyed by beta power consistently preceded that of alpha and gamma with a median difference in $\tau$ of $0.5 \mathrm{~s}$ both when comparing the information in beta and gamma and that in beta and alpha $(p<0.05$, Wilcoxon test, uncorrected).

We considered whether the amplitude of a frequency component of the neural signal predicted the information it carries about BOLD. We did not find significant correlation $(p>0.05, t$ test) between LFP power and the information values at $\tau=3 \mathrm{~s}$ for any frequency of the LFP spectrum. This suggests that strength of the relationship between the power at a given frequency and the BOLD signal cannot be predicted based on the relative power of the LFP spectrum at that frequency.

To understand whether the power in the LFP bands and the BOLD signal covary or anti-covary we computed the Pearson correlation coefficient between the two signals. We found positive correlation (Figs. $2 B$, inset, $3 B$, inset) for all LFP bands, indicating that an increase in the power of any of the bands corresponds to an increase in the amplitude of the BOLD signal. The correlation plots confirmed that changes in beta power are reflected earlier by changes occurring in the BOLD signal compared with the other bands considered. However, we observed that while gamma information was approximately twofold, the information carried by beta power, the ratio between the gamma and the beta correlation coefficients, was only equal to 1.6 . Because Pearson correlation quantifies only the linear part of the relationship between the power of an LFP band and the BOLD signal (while mutual information takes into account all possible types of relationship), this observation suggests that a linear model may not fully capture the relationship between gamma power and the BOLD signal.

Finally, we considered MUA. The temporal profile of information about BOLD carried by MUA was similar to that of the gamma and alpha bands (Figs. $2 B, 3 B$ ). Consistent with previous work (Logothetis et al., 2001; Kayser et al., 2004; Niessing et al., 2005; Murayama et al., 2010), the information conveyed by MUA about hemodynamic activity (median, 0.017 bits; 40th to 60th percentile range, $0.012-0.019$ bits; uncorrected) was lower than that carried by gamma power, suggesting that LFPs are the primary neural correlate of the BOLD signal. In the following, we will therefore concentrate on the LFP frequency range and investigate in detail how the different components of its spectrum reflect in the BOLD signal.

The amplitude of the BOLD signal scales with gamma power The high information values between gamma power and the BOLD signal indicate, consistently with previous studies (Logothetis et al., 2001, 2010; Kayser et al., 2004; Niessing et al., 2005; Goense and Logothetis, 2008; Murayama et al., 2010; Schölvinck et al., 2010), that the gamma band is the LFP band that more strongly relates to changes in the BOLD signal. Information, however, does not tell us about the form of the relationship existing between the BOLD signal and gamma power. To understand how changes in gamma power are related to changes in the BOLD signal, we thus characterized the specific variations in BOLD signal following changes in gamma power as follows. We subdivided the values of gamma power in each session into 10 percentile levels, and we computed, for each level, the corresponding variation in BOLD signal. The procedure is illustrated in Figure $4 A$ for the highest (top 10\%) gamma power level considered and for a representative session (I02tv1).

We observed (Fig. $4 B$ ) that we could subdivide the gamma power into three groups based on whether the BOLD signal increased, decreased, or did not change following the selected 

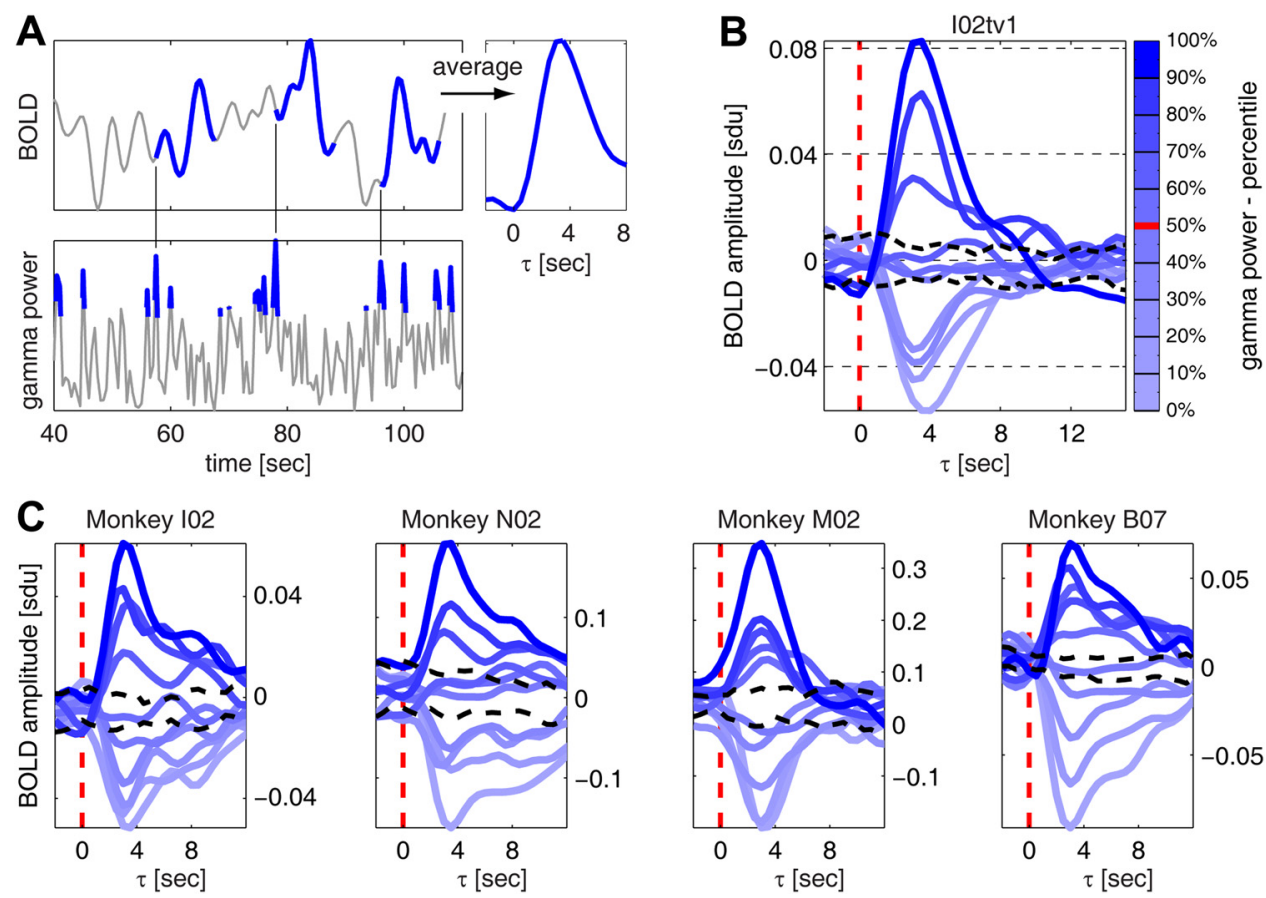

Figure 4. The amplitude of the BOLD signal scales with gamma power. $A$, Illustration of the procedure for computing average changes in the BOLD signal following different intensities of gamma power. Data are from session 102tv1. Left, The top 10\% gamma power data points and the BOLD signal in the 15 s following three of the detected high-gamma power events are highlighted in blue. Right, Average change in BOLD signal following the top $10 \%$ gamma power data points. $\boldsymbol{B}$, Average changes in BOLD signal for each of the 10 percentile levels of gamma power (same data as in $\boldsymbol{A}$ ). The significance levels (black dashed lines) were computed as the fifth and 95 th percentile of the distribution of changes in BOLD signal following sets of randomly selected data points (the number of points in each random set being equal to the number of points in each of the 10 gamma percentile levels). $\boldsymbol{C}$, Average changes in BOLD signal (same type of plot as in $\boldsymbol{B}$ ) for four additional sessions. One representative session is shown for each monkey.

gamma power events. On average, the BOLD signal increased significantly $(p<0.05$; bootstrap test is described in the caption of Fig. 4) following gamma power in the top three percentile levels (covering the range $70-100 \%$ ), while it decreased significantly $(p<0.05)$ following gamma power in the lowest four levels considered (range $0-40 \%$ ). A decrease in gamma power below its median value (Fig. $4 B$, red mark in the colorbar) led to a decrease in BOLD signal of magnitude comparable to the increases observed following the top three percentile gamma levels. Finally, when gamma power was close to its median value (40-70\% range) changes in BOLD fMRI were not significant.

We observed that the amplitude of the peaks and troughs of the computed changes in BOLD signal scaled with gamma power intensity. The higher the gamma power, the higher the peak of the subsequent BOLD signal increase. The lower the gamma power, the lower the trough of the BOLD signal decrease. Moreover, both peaks and troughs occurred with a $3.5 \mathrm{~s}$ delay. These results indicate that increases and decreases in gamma power can predict as reliably both increases and decreases in the BOLD signal that occur a few seconds later.

Our measures of the maximally informative delays between the neural activity and the BOLD signal are in agreement with data from combined neurophysiological BOLD fMRI recordings in anesthetized monkeys during spontaneous activity previously reported from our laboratory (Murayama et al., 2010). In this study, using different analysis techniques, we found maximal neurovascular coupling at a lag of 4-5 s. The small difference in time lags between the current study and the earlier one may be attributed to the longer intervolume time (2s) used in the earlier study, compared with the high temporal resolution (intervolume time $=0.5 \mathrm{~s}$ ) used in our current experiments. We verified that if we downsampled our data with 2 s intervals, we obtained information and correlation peaks with a delay of $4 \mathrm{~s}$ (data not shown).

The above results were highly consistent across sessions and monkeys (Fig. 4C). To investigate quantitatively the scaling of the amplitude of BOLD signal changes with gamma power at the population level, we computed the difference in average BOLD amplitude between each gamma percentile level and the percentile level immediately below at a lag $\tau=3.5 \mathrm{~s}$. We then pooled the difference values for all percentile levels and sessions, and found that at the population level these average differences were significantly positive ( $p<10^{-10}, t$ test; data not shown).

\section{Beta and alpha power carry information complementary to that of gamma power}

The above information theoretical analysis revealed that, although the gamma band was the one with the highest information, also the alpha and beta bands conveyed information about the BOLD signal. An important question is whether these two bands merely reflect information already contained in gamma power or whether they instead carry information, which is of a different nature with respect to that in gamma power.

To address this question, we quantified the difference between the information increase obtained when combining gamma power, together with the power in either the beta or alpha band, and the information carried by gamma power alone. This information gain corresponds to the amount of complementary information carried by the alpha or beta band over the information carried by the gamma band (see Materials and Methods).

We found (Fig. 5A) that the beta band added the largest amount of information complementary to that carried by the gamma band. When combining beta power with gamma power, 
the complementary information was higher between 1.5 and $3.5 \mathrm{~s}$. Over this time range, the median information increase was significant $(p<0.05$, bootstrap test, uncorrected) and was 0.006 bits (interquartile range, 0.004-0.015 bits), which corresponds to a gain of $\sim 30 \%$ over the information conveyed by gamma power alone (Fig. $5 B$ ).

The complementarity of information between alpha and gamma power (Fig. $5 A$ ) occurred between 2.5 and 4 s. The amount of complementary information carried by alpha power over this time range was significant $(p<0.05$, bootstrap test, uncorrected) and had a median value of 0.002 bits (interquartile range, $0.001-$ 0.008 bits), which corresponds to a $7 \%$ gain in information (Fig. $5 B$ ). The information gain obtained for alpha power was significantly smaller than that obtained for beta power $(p<0.01$, Wilcoxon rank sum test).

\section{The information added specifically by beta power}

The high values of complementary information observed between gamma and beta power indicate that beta power can be used to improve the prediction about the BOLD signal obtained using gamma power alone. To understand what information is added specifically by beta power, we investigated the relationship between beta power and the BOLD signal when variations in gamma power were limited to a small range of intensities. To this aim, we subdivided gamma power into three percentile levelslow, median, and high gamma power-approximately corresponding to the three ranges of intensities that were found above to be followed by decreases, no change, or increases in BOLD signal.

We begin by considering the relationship between beta power and the BOLD signal when gamma power was within the high (top 33\%) percentile range. We selected values of beta power corresponding to gamma power within this high range of intensities, we subdivided them into three percentile levels-low, median, and high beta power-and then computed the average variation in BOLD signal following each level. This procedure is illustrated in Figure $6 \mathrm{~A}$ for a representative session (I02tv1). On average, the BOLD signal exhibited an increase following all three levels of beta considered (Fig. $6 \mathrm{~B}$ ), as expected from the fact that we considered high values of gamma power. However, we found that between 0.5 and $3.5 \mathrm{~s}$ (highlighted in the inset) the shape of the three BOLD curves could be discriminated based on beta power. In particular, BOLD signal increases were anticipated or delayed depending on whether the accompanying beta power was high or low, respectively. While this observation is consistent with and can partially explain the earlier onset of information (and Pearson correlation) observed for the beta band in Figures 2 and 3 , it is important to note that this timing relationship could not have been predicted based solely on the results of an information or correlation analysis.

To confirm this effect at the population level, we increased the sampling rate by linear interpolation and computed the difference in rising time (defined as the lag at which the average BOLD change reached the middle point between the value at $\tau=0$ and the peak) between neighboring levels of beta power. We found (Fig. 6D) that, over the population, increasing beta power by one percentile level shifted the rising time by $-0.2 \mathrm{~s}$ (median value; interquartile range -0.35 to $-0.1 ; p<10^{-6}, t$ test), indicating that high beta power corresponds to an anticipation of the variations in the BOLD signal following high-gamma power events.

To confirm that the time shift observed in the BOLD signal changes was indeed characteristic of the beta band and not just of low frequencies in general, we performed the same analysis using frequency ranges other than the beta band. We found (Fig. 6C,D) that using alpha power, instead of beta, did not produce any shift in the time of increases in the BOLD signal following high values of gamma power ( $p=0.35, t$ test). Moreover, we repeated the analysis using gamma power itself as a modulating factor (i.e., we further subdivided the top $33 \%$ gamma power values into three gamma sublevels). We also found (Fig. 6D) that in this case the distribution of the differences in rising time was not significantly different from zero ( $p=0.13, t$ test, uncorrected $)$, suggesting that the observed beta power-related time shifts in the BOLD signal responses to high gamma power activity cannot be explained by inhomogeneities in the values of gamma power within a class. We only observed a significant time shift of the BOLD signal following high gamma activity when conditioning the responses upon the total $(0-100 \mathrm{~Hz})$ LFP power instead of beta power (Fig. 6D) ( $p=0.015, t$ test, uncorrected). However, this result could be explained simply with the observation that the total LFP power also includes beta power. When conditioning the changes in the BOLD signal to gamma activity upon the power in the total LFP band minus the power in the beta band (Fig. $6 D$ ), the shift in the rising times was no longer significant $(p=0.24$, $t$ test, uncorrected).

To understand how beta power reflects changes in decreases in BOLD signal following low values of gamma power, we investigated the relationship between beta power and the BOLD signal when gamma power was within its lowest $33 \%$ range. The results are shown in the bottom plot of Figure $6 \mathrm{~B}$. For all levels of beta power, the BOLD signal exhibited on average a decrease in amplitude, as expected from the fact that we considered the lowest 
A

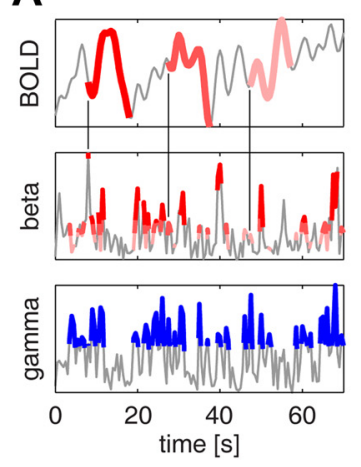

B

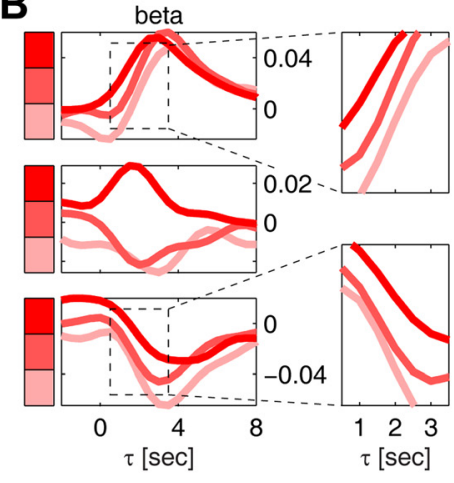

D Lag of BOLD Increase

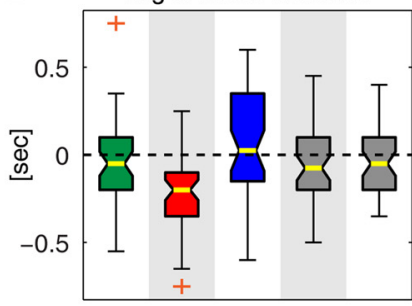

Lag of BOLD Decrease

C
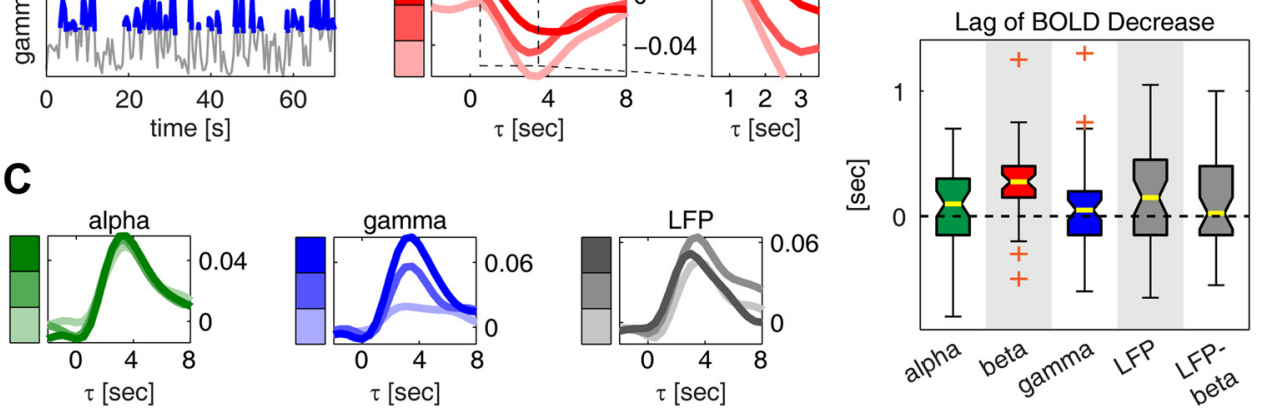

Figure 6. Mechanisms of the complementarity between gamma and beta power. $\boldsymbol{A}$, Illustration of the procedure for computing average changes in BOLD signal following different intensities of beta power at fixed gamma power. Data are from session 102tv1. Gamma power data points corresponding to the top 33\% gamma power intensities are marked in blue. Beta power values during these epochs of high gamma power are subdivided into three equally populated levels of increasing intensity highlighted with three shades of red (lighter red corresponds to lower beta intensities). Changes in BOLD signal in the $15 \mathrm{~s}$ following detected beta power data points are indicated using the same color convention as for beta power. Only three BOLD segments are shown for simplicity, one segment for each level of beta power. $B$, Average changes in BOLD signal following each of the three levels of beta power at fixed gamma power. Same data as in $A$. Results are shown for each of the three fixed gamma levels (top, middle, and lowest 33\%). The insets show a detail of the average BOLD curves between 1.5 and $3.5 \mathrm{~s}$. $\boldsymbol{C}$, Same plot as in $\boldsymbol{B}$, top, but for the average BOLD changes computed using alpha power (green), gamma power (blue), and the total LFP ( $0-100 \mathrm{~Hz}$ ) power (gray) instead of beta power. $D$, Distribution of the shift of rising time between neighboring levels of beta power (see main text). Box plots are shown also for the alpha, gamma, total LFP power, and the difference, LFP-beta, between the total LFP power and the beta band. Results are plotted as median (yellow bar) and interquartile range (box). The whiskers extend to the most extreme data points not considered outliers, and outliers are plotted as orange crosses. Bands for which the distribution of the rising time was significantly negative ( $p<0.05$, Wilcoxon signed rank test) are highlighted in gray. The top (bottom) plot shows the shifts in rising time of the BOLD responses following the top (lowest) 33\% of gamma power events. Negative (positive) shifts in rising time mean anticipation (delay) of the variations in the BOLD signal following the high (low) gamma power event.

gamma power intensities. Again, we observed that between 0.5 and $3.5 \mathrm{~s}$ (highlighted in the inset) the average BOLD signal curves could be discriminated based on beta power. In this case, however, decreases in the BOLD signal occurred later for higher values of beta power. We tested this result at the population level in a similar way to what we did for the increasing BOLD curves. Again, we found that only when using beta power the shifts in the average BOLD signal changes were significant $(p<0.05, t$ test, uncorrected).

Finally, we observed no relationship between beta power and the BOLD signal when gamma power was in the middle $33 \%$ level, consistent with the observation that no significant changes in BOLD signal occurred when gamma power was within this range.

\section{How the relationship between gamma power and alpha power reflects upon the BOLD signal}

We next investigated the nature of the relationship between the respective contribution of alpha and gamma to the BOLD signal. A recent theory (Kilner et al., 2005) suggests that increases in the BOLD response are associated with a loss of power in low LFP frequencies (e.g., the alpha band) relative to high frequencies (e.g., the gamma band). The heuristic described by Kilner et al. (2005) is based upon the straightforward idea that neuronal activation is mediated by an increase in coupling among neuronal populations. In dynamic systems, a coupling corresponds to a rate constant, which means that activations are associated with increased rate constants and faster dynamics; in other words, a shift in the frequency profile toward higher frequencies. Neurophysiologically, this is intuitive because increased synaptic activ- ity increases postsynaptic membrane conductance and therefore decreases the effective membrane time constants. In other words, this theory states that the BOLD signal does not depend only on a frequency band per se but rather on the overall spectral profile, with spectral profiles biased toward higher frequencies giving the largest BOLD response. A simple prediction of this theory is that an increase in low-frequency power (e.g., alpha) without a change in total LFP power would lead to a reduction in BOLD signal, whereas an increase in high-frequency power (e.g., gamma) without a change in total LFP power would increase the BOLD signal.

We tested this prediction by evaluating the relationship between each frequency in the LFP spectrum and the BOLD signal at fixed values of the total LFP power. We subdivided the total $(0-100 \mathrm{~Hz})$ LFP power into 10 percentile levels and computed, for each level, the Pearson correlation coefficient between LFP power at each frequency and the BOLD signal at different lags. For this analysis, we used Pearson correlation because, unlike information, it can assume both positive and negative values, thus indicating whether the signals covary or anti-covary. We found that, while overall (i.e., across all data points) the correlation between single LFP frequencies and the BOLD signal was positive for all LFP frequencies (Figs. $3 B, 7 A$ ), when considering fixed levels of total LFP power the Pearson correlation was negative for frequencies $<20 \mathrm{~Hz}$, reaching a minimum at $12 \mathrm{~Hz}$. The correlation remained instead positive for frequencies $>30 \mathrm{~Hz}$. These results are compatible with the prediction of the model proposed by Kilner et al. (2005). Additionally, these results establish a clear frequency boundary at $\sim 20 \mathrm{~Hz}$ for identifying the 


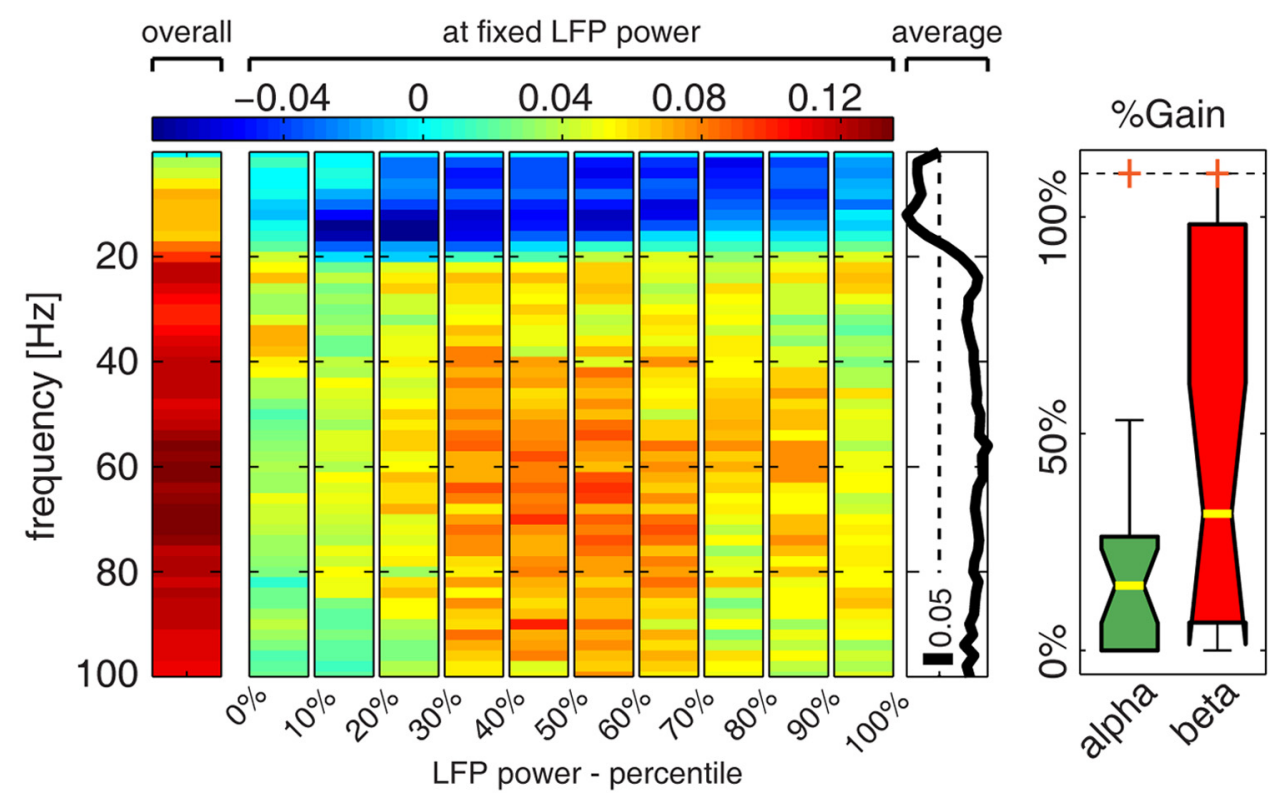

Figure 7. Alpha power and the BOLD signal anticorrelate at fixed LFP power. A, Pearson correlation between the power at single LFP frequencies and the BOLD signal delayed by $3.5 \mathrm{~s}$ with respect to the electrophysiology. The left plot shows the overall correlation coefficient computed across all data points. The middle plot shows the Pearson correlation computed only across data points belonging to the LFP power percentile indicated on the $x$-axis (we call this "correlation at fixed LFP power"). The right plot shows the average of the Pearson correlation at fixed LFP power over all LFP percentile levels. $\boldsymbol{B}$, Percentage information gain yielded by alpha and beta power over the information conveyed by gamma power at fixed total LFP power (gain values were averaged over the 10 percentile levels in which the total LFP power was subdivided). Results are plotted as median (yellow bar) and interquartile range (box). The whiskers extend to the most extreme data points not considered outliers, and outliers are plotted as orange crosses (outliers outside the range $0-110 \%$ are moved to these limits).

range of low frequencies that anticorrelate with the BOLD signal at fixed total LFP power.

We next investigated whether the difference in sign of the correlation between alpha or gamma power and the BOLD signal at fixed total LFP power reflects a genuine dependency of the BOLD signal upon the relative power of the gamma and alpha bands, as it would be predicted by the model in Kilner et al. (2005). We tested this by computing the complementarity of the information about the BOLD signal conveyed by gamma and alpha at fixed LFP power. We found that, at fixed LFP power, knowledge of alpha power increased by $15 \%$ the amount of information about the BOLD signal that could be extracted from gamma power alone (Fig. $7 B$ ). The fact that alpha power adds information that cannot possibly be extracted from gamma power means that the BOLD signal genuinely correlates with the relative spectral profile of alpha and gamma power beyond what can be explained by the relation between BOLD and total LFP power or between BOLD and the power in a single band.

The complementarity of information between alpha and gamma power was less pronounced when considering all data points (Fig. 5B) rather than when considering data points at fixed total LFP power (Fig. 7B). In our view, this was at least partly due to covariations between the LFP bands. We found that the power in the alpha, beta, and gamma bands covaried across trials following time variations of the total power. The degree of covariation between alpha and gamma may be a factor reducing their overall complementarity.

It is worth noting that the correlation between gamma and beta is expected to have little or no impact upon the complementarity between gamma and beta, because shift in the timing of the BOLD signal changes following variations of gamma power response was found to be characteristic of beta power only and does not depend on the total LFP (once beta power has been removed) (Fig. $6 D$ ). Indeed, our analysis shows that the degree of complementarity of information about BOLD between gamma and beta power was the same overall (Fig. 5B) and at fixed total LFP power (Fig. 7B).

In sum, our analysis suggests a genuine dependency of the BOLD response amplitude upon the overall shape of the LFP spectrum, in particular with the BOLD response amplitude being sensitive to the conjunction of values of alpha and gamma power rather than being exclusively sensitive either to the total LFP power or to the power of a single band.

\section{Discussion}

Substantial evidence suggests that components of mass neural activity that contribute to the LFP can be separated, to a large extent, by decomposing it into different nonoverlapping frequency regions. Therefore, characterizing the relationship between BOLD contrast changes and different frequency regions of the LFP spectrum is useful for understanding what fMRI can tell us about the involvement of different neural pathways to cortical processing. Here we made progress in this direction by using a combination of simultaneous recordings of LFP and fMRI during spontaneous activity with mathematical analyses to investigate whether the BOLD signal reflects specific relationships among different LFP bands. These data were collected under remifentanil anesthesia because it allows recording of large datasets while minimally affecting both neurophysiological and fMRI responses, as well as their coupling, in primary visual cortex (Goense and Logothetis, 2008). We found a particularly strong relationship between BOLD and LFP frequencies in the range between 8 and $100 \mathrm{~Hz}$. The relative values of the power across different parts of the spectrum affected both the timing and the 
amplitude of the BOLD signal. The significance of our findings is discussed in the following.

\section{The amplitude of the BOLD signal reliably reflects both increases and decreases in gamma power}

Consistent with previous studies (Logothetis et al., 2001, 2010; Kayser et al., 2004; Niessing et al., 2005; Goense and Logothetis, 2008; Murayama et al., 2010; Schölvinck et al., 2010), we found that the LFP frequency range that was most informative about the BOLD signal was the $40-100 \mathrm{~Hz}$ gamma range. The LFP power in this frequency range has stimulus tuning properties that are partly different from those of spiking activity (Gieselmann and Thiele, 2008; Ray and Maunsell, 2011) and have been hypothesized to reflect recurrent interactions between inhibitory and excitatory cortical neurons (Brunel and Wang, 2003). Although in principle the information between the BOLD contrast and gamma power could result from reliable changes in BOLD signal only in response to increases of gamma activity and not to its decreases, here we demonstrated that this was not the case: changes in BOLD signal followed reliably both increases and decreases in gamma power. This shows that the neural deactivation reflected in a decrease of gamma power can be indeed mapped not only from sustained negative BOLD responses induced by specifically designed stimulation (Shmuel et al., 2006) but also from subtle, single-trial variations such as those observed during spontaneous activity.

\section{Timing of variations of the BOLD signal reflect activity in the beta band}

We found that the $18-30 \mathrm{~Hz}$ beta range was the second most informative LFP band, carrying information complementary to that of the gamma band. This is consistent with a previous study, which suggested an independent contribution of beta $(18-28 \mathrm{~Hz})$ and gamma power to the BOLD variance (Scheeringa et al., 2011). Our data provided novel insights into the nature of this contribution by revealing that beta power correlates with how fast changes in the BOLD signal occur following changes in gamma power. Higher beta power corresponds to faster increases (slower decreases) of the BOLD signal, and lower beta power corresponds to faster decreases (and slower increases) in BOLD signal.

Second, our demonstration of complementarity using information theory represents itself a methodological advance. Because information captures all ways by which a signal relates to another, finding that another signal carries extra information demonstrates that this signal truly provides some information that cannot be possibly obtained from the first one. This does not necessarily hold when using methods that capture only specific relationships between signals. For example, an increase in predictability based on linear models may reflect both additional information from the second regressor as well as information that was already present in the first regressor but was not captured by the linear assumption.

In a previous study (Belitski et al., 2008) in the same cortical areas and under the same anesthesia conditions, we reported that, while the gamma band was strongly stimulus modulated, the frequencies in the beta range, though highly correlated between each other and so likely arising mostly from a single source, were largely stimulus independent. This led us to suggest that the beta band may reflect the contribution of a stimulus-independent neuromodulatory pathway, and we therefore named this frequency range the "neuromodulatory band" (Belitski et al., 2008). Release of neuromodulators such as acetylcholine, noradrena- line, and serotonin has been shown to affect cortical excitability (Steriade et al., 1993). Neuromodulators may alter the relationship between the BOLD signal and neural activity in many ways, for example, by affecting key neural parameters such as the balance between excitation and inhibition (Constantinople and Bruno, 2011), or they may be involved in the regulation of cerebral blood flow and neurovascular coupling (Girouard and Iadecola, 2006; Drake and Iadecola, 2007), therefore allowing faster or slower hemodynamic responses. An important caveat is that the statistical relationship observed between beta power and the BOLD signal does not necessarily imply causality from beta power to BOLD but could reflect, instead, coupling between different LFP bands. For example, beta power may precede increases in gamma power that are reported by BOLD slightly later in time. Nonlinear coupling of this type is implicit in the neuromodulatory correlates of beta activity discussed above and will need further work to be comprehensively documented, using, for example, a Volterra description (Friston et al., 2000) of these interactions.

Cortical microcircuits are thought to be capable of changing their mode of operation by acting either as drivers, faithfully transmitting stimulus-related information, or as modulators, adjusting the overall sensitivity and context specificity of the responses. Being able to track the relationship between these modes of operation and BOLD responses would greatly enhance our understanding of how to differentiate between function-specific processing and neuromodulation in BOLD fMRI. The results presented here make some steps in this direction by suggesting that it might be possible to separately map stimulus-related activity (e.g., gamma) and stimulus-unrelated activity (e.g., beta) using the BOLD signal, given that these components of neural activity are to some extent reflected differentially in the BOLD contrast.

\section{The amplitude of the BOLD signal and the relative power of high- and low-frequency neural activity: implications for models of neurovascular coupling}

Recent theoretical work suggested that the amplitude of the BOLD signal may depend on the ratio between the power of neural activity at low and high frequencies, because the energy dissipation of neural activity may be more pronounced at higher frequencies (Kilner et al., 2005). This model was recently tested on simultaneous EEG-fMRI recordings with contrasting results and interpretations (Rosa et al., 2010; Scheeringa et al., 2011). Our results provide a direct confirmation, based on simultaneous colocalized electrophysiological and fMRI recordings, of the hypotheses of Kilner et al. (2005), as we showed that an increase of LFP power in the range $8-12 \mathrm{~Hz}$, referred to as the alpha band, without a change in total LFP power led to a reduction in the BOLD response, whereas an increase in gamma power without a change in total LFP power increased the BOLD activation. Moreover, our result that alpha power carried information about BOLD complementary to that of gamma power (both at fixed total LFP power and across all data points) further supports the view that the amplitude of the BOLD response genuinely depends upon the overall shape of the LFP spectrum and not simply upon the total LFP power or the power of a single band.

Our results should not be interpreted as showing that the BOLD signal only or primarily reflects the ratio between the power at low and high frequencies, but rather that the relative power across frequencies is one of the factors modulating the relationship between the BOLD signal and neural power. In our view, the report by Scheeringa et al. (2011) of negligible trial-to- 
trial covariations of gamma and alpha power at fixed BOLD signal amplitude is also compatible with the considerations of Kilner et al. (2005) and with the results reported here. In fact, a given value of BOLD amplitude may, for example, be obtained either with high total LFP power and relative high alpha power, or with lower total LFP power but relative high gamma power. This may result in overall weak trial-to-trial correlations between alpha and gamma power at fixed BOLD values.

Previous studies revealed both positive (Goense and Logothetis, 2008; Murayama et al., 2010) and negative (Mukamel et al., 2005; Schölvinck et al., 2010) correlation values between alpha power and BOLD fMRI. This difference may be partly reconciled by our finding that both alpha power and the BOLD signal were positively correlated with the total LFP $(0-100 \mathrm{~Hz})$ power. When large variations of total LFP power occur, alpha power will appear to be positively correlated with the BOLD signal as a reflection of their common covariation with total LFP power. However, when the range of variations of total LFP power within the experiment is restricted, for example, by fixing the stimulus or the task, then alpha power may also be anticorrelated with the BOLD signal.

\section{References}

Basar E (1980) EEG-brain dynamics: relation between EEG and brain evoked potentials. Amsterdam: Elsevier-North-Holland Biomedical.

Belitski A, Gretton A, Magri C, Murayama Y, Montemurro MA, Logothetis NK, Panzeri S (2008) Low-frequency local field potentials and spikes in primary visual cortex convey independent visual information. J Neurosci 28:5696-5709.

Brown GG, Perthen JE, Liu TT, Buxton RB (2007) A primer on functional magnetic resonance imaging. Neuropsychol Rev 17:107-125.

Brunel N, Wang XJ (2003) What determines the frequency of fast network oscillations with irregular neural discharges? I. Synaptic dynamics and excitation-inhibition balance. J Neurophysiol 90:415-430.

Constantinople CM, Bruno RM (2011) Effects and mechanisms of wakefulness on local cortical networks. Neuron 69:1061-1068.

Creutzfeldt OD, Watanabe S, Lux HD (1966a) Relations between EEG phenomena and potentials of single cortical cells. I. Evoked responses after thalamic and epicortical stimulation. Electroencephalogr Clin Neurophysiol 20:1-18.

Creutzfeldt OD, Watanabe S, Lux HD (1966b) Relations between EEG phenomena and potentials of single cortical cells. II. Spontaneous and convulsoid activity. Electroencephalogr Clin Neurophysiol 20:19-37.

D'Esposito M, Deouell LY, Gazzaley A (2003) Alterations in the BOLD fMRI signal with ageing and disease: a challenge for neuroimaging. Nat Rev Neurosci 4:863-872.

Drake CT, Iadecola C (2007) The role of neuronal signaling in controlling cerebral blood flow. Brain Lang 102:141-152.

Friston KJ, Holmes AP, Worsley KJ, Poline JP, Frith CD, Frackowiak RSJ (1994) Statistical parametric maps in functional imaging: a general linear approach. Hum Brain Mapp 2:189-210.

Friston KJ, Mechelli A, Turner R, Price CJ (2000) Nonlinear responses in fMRI: the Balloon model, Volterra kernels, and other hemodynamics. Neuroimage 12:466-477.

Fuhrmann Alpert G, Sun FT, Handwerker D, D’Esposito M, Knight RT (2007) Spatio-temporal information analysis of event-related BOLD responses. Neuroimage 34:1545-1561.

Gieselmann MA, Thiele A (2008) Comparison of spatial integration and surround suppression characteristics in spiking activity and the local field potential in macaque V1. Eur J Neurosci 28:447-459.

Girouard H, Iadecola C (2006) Neurovascular coupling in the normal brain and in hypertension, stroke, and Alzheimer disease. J Appl Physiol 100:328-335.

Goense JB, Logothetis NK (2008) Neurophysiology of the BOLD fMRI signal in awake monkeys. Curr Biol 18:631-640.

Goense JB, Ku SP, Merkle H, Tolias AS, Logothetis NK (2008) fMRI of the temporal lobe of the awake monkey at 7 T. Neuroimage 39:1081-1093.

Granit R, Kernell D, Smith R (1963) Delayed depolarization and the repetitive response to intracellular stimulation of mammalian motoneurones. J Physiol 168:890-910.
Haase A, Frahm J, Matthaei D, Hanicke W, Merboldt KD (1986) FLASH imaging. Rapid NMR imaging using low flip-angle pulses. J Magn Reson 67:258-266.

Jones AK, Qi LY, Fujirawa T, Luthra SK, Ashburner J, Bloomfield P, Cunningham VJ, Itoh M, Fukuda H, Jones T (1991) In vivo distribution of opioid receptors in man in relation to the cortical projections of the medial and lateral pain systems measured with positron emission tomography. Neurosci Lett 126:25-28.

Juergens E, Guettler A, Eckhorn R (1999) Visual stimulation elicits locked and induced gamma oscillations in monkey intracortical-and EEGpotentials, but not in human EEG. Exp Brain Res 129:247-259.

Kamondi A, Acsády L, Wang XJ, Buzsáki G (1998) Theta oscillations in somata and dendrites of hippocampal pyramidal cells in vivo: activity dependent phase precession of action potentials. Hippocampus 8:244-261.

Kayser C, Kim M, Ugurbil K, Kim DS, König P (2004) A comparison of hemodynamic and neural responses in cat visual cortex using complex stimuli. Cereb Cortex 14:881-891.

Kilner JM, Mattout J, Henson R, Friston KJ (2005) Hemodynamic correlates of EEG: a heuristic. Neuroimage 28:280-286.

Klee MR, Offenloch K, Tigges J (1965) Cross-correlation analysis of electroencephalographic potentials and slow membrane transients. Science 147:519-521.

Lindsley DB, Wicke J (1974) The electroencephalogram: autonomous electrical activity in man and animals. In: Bioelectric recording techniques: Part B: Electroencephalography and human brain potentials (Thompson RF, Patterson MM, eds), pp 3-83. New York: Academic.

Logothetis NK (2003) The underpinnings of the BOLD functional magnetic resonance imaging signal. J Neurosci 23:3963-3971.

Logothetis NK (2008) What we can do and what we cannot do with fMRI Nature 453:869-878.

Logothetis NK, Guggenberger H, Peled S, Pauls J (1999) Functional imaging of the monkey brain. Nat Neurosci 2:555-562.

Logothetis NK, Pauls J, Augath M, Trinath T, Oeltermann A (2001) Neurophysiological investigation of the basis of the fMRI signal. Nature 412:150-157.

Logothetis NK, Murayama Y, Augath M, Steffen T, Werner J, Oeltermann A (2009) How not to study spontaneous activity. Neuroimage 45:1080-1089.

Logothetis NK, Augath M, Murayama Y, Rauch A, Sultan F, Goense J, Oeltermann A, Merkle H (2010) The effects of electrical microstimulation on cortical signal propagation. Nat Neurosci 13:1283-1291.

Lund JS, Yoshioka T, Levitt J (1994) Substrates for interlaminar connections in area VI of macaque monkey cerebral cortex. In: Cerebral cortex: primary visual cortex in primates, Vol 10 (Peters AA, Rockland KS, eds), pp 37-60. New York: Plenum.

Mitzdorf U (1985) Current source-density method and application in cat cerebral cortex: investigation of evoked potentials and EEG phenomena. Physiol Rev 65:37-100.

Mitzdorf U (1987) Properties of the evoked potential generators: current source-density analysis of visually evoked potentials in the cat cortex. Int J Neurosci 33:33-59.

Mukamel R, Gelbard H, Arieli A, Hasson U, Fried I, Malach R (2005) Coupling between neuronal firing, field potentials, and FMRI in human auditory cortex. Science 309:951-954.

Murakami S, Okada Y (2006) Contributions of principal neocortical neurons to magnetoencephalography and electroencephalography signals. J Physiol 575:925-936.

Murayama Y, Biessmann F, Meinecke FC, Müller KR, Augath M, Oeltermann A, Logothetis NK (2010) Relationship between neural and hemodynamic signals during spontaneous activity studied with temporal kernel CCA. Magn Reson Imaging 28:1095-1103.

Nicholson C (1973) Theoretical analysis of field potentials in anisotropic ensembles of neuronal elements. IEEE Trans Biomed Eng 20:278-288.

Niessing J, Ebisch B, Schmidt KE, Niessing M, Singer W, Galuske RA (2005) Hemodynamic signals correlate tightly with synchronized gamma oscillations. Science 309:948-951.

Oeltermann A, Augath MA, Logothetis NK (2007) Simultaneous recording of neuronal signals and functional NMR imaging. Magn Reson Imaging 25:760-774.

Ogawa S, Tank DW, Menon R, Ellermann JM, Kim SG, Merkle H, Ugurbil K (1992) Intrinsic signal changes accompanying sensory stimulation: func- 
tional brain mapping with magnetic resonance imaging. Proc Natl Acad Sci U S A 89:5951-5955.

Panzeri S, Treves A (1996) Analytical estimates of limited sampling biases in different information measures. Netw Comput Neural Syst 7:87-108.

Panzeri S, Senatore R, Montemurro MA, Petersen RS (2007) Correcting for the sampling bias problem in spike train information measures. J Neurophysiol 98:1064-1072.

Percival DB, Walden AT (1993) Spectral analysis for physical applications: multitaper and conventional univariate techniques. Cambridge, UK: Cambridge UP.

Pettersen KH, Devor A, Ulbert I, Dale AM, Einevoll GT (2006) Currentsource density estimation based on inversion of electrostatic forward solution: effects of finite extent of neuronal activity and conductivity discontinuities. J Neurosci Methods 154:116-133.

Rauch A, Rainer G, Logothetis NK (2008) The effect of a serotonin-induced dissociation between spiking and perisynaptic activity on BOLD functional MRI. Proc Natl Acad Sci U S A 105:6759-6764.

Ray S, Maunsell JH (2011) Different Origins of Gamma Rhythm and HighGamma Activity in Macaque Visual Cortex. PLoS Biology 9:e1000610.

Rosa MJ, Kilner J, Blankenburg F, Josephs O, Penny W (2010) Estimating the transfer function from neuronal activity to BOLD using simultaneous EEG-fMRI. Neuroimage 49:1496-1509.

Saleem K, Logothetis N (2007) Atlas of the rhesus monkey brain. London: Elsevier.

Scheeringa R, Fries P, Petersson KM, Oostenveld R, Grothe I, Norris DG, Hagoort P, Bastiaansen MC (2011) Neuronal dynamics underlying high-and low-frequency eeg oscillations contribute independently to the human BOLD signal. Neuron 69:572-583.

Schölvinck ML, Maier A, Ye FQ, Duyn JH, Leopold DA (2010) Neural basis of global resting-state fMRI activity. Proc Natl Acad Sci U S A 107:10238-10243.

Schroeder CE, Steinschneider M, Javitt DC, Tenke CE, Givre SJ, Mehta AD, Simpson GV, Arezzo JC, Vaughan HG Jr (1995) Localization of ERP generators and identification of underlying neural processes. Electroencephalogr Clin Neurophysiol Suppl 44:55-75.

Schroeder CE, Mehta AD, Givre SJ (1998) A spatiotemporal profile of visual system activation revealed by current source density analysis in the awake macaque. Cereb Cortex 8:575-592.

Sereno ME, Trinath T, Augath M, Logothetis NK (2002) Three-dimensional shape representation in monkey cortex. Neuron 33:635-652.

Shannon CE (1948) A mathematical theory of communication. Bell System Technical J 27:623-656.

Shmuel A, Augath M, Oeltermann A, Logothetis NK (2006) Negative functional MRI response correlates with decreases in neuronal activity in monkey visual area V1. Nat Neurosci 9:569-577.

Steriade M, Hobson J (1976) Neuronal activity during the sleep-waking cycle. Prog Neurobiol 6:155-376.

Steriade M, McCormick DA, Sejnowski TJ (1993) Thalamocortical oscillations in the sleeping and aroused brain. Science 262:679-685.

Thomsen K, Offenhauser N, Lauritzen M (2004) Principal neuron spiking: neither necessary nor sufficient for cerebral blood flow in rat cerebellum. J Physiol 560:181-189.

Thomson DJ (1982) Spectrum estimation and harmonic analysis. Proc IEEE 70:1055-1096.

Viswanathan A, Freeman RD (2007) Neurometabolic coupling in cerebral cortex reflects synaptic more than spiking activity. Nat Neurosci 10:1308-1312.

Worsley KJ, Friston KJ (1995) Analysis of fMRI time-series revisitedagain. Neuroimage 2:173-181.

Zappe AC, Uludağ K, Oeltermann A, Uğurbil K, Logothetis NK (2008a) The influence of moderate hypercapnia on neural activity in the anesthetized nonhuman primate. Cereb Cortex 18:2666-2673.

Zappe AC, Uludağ K, Logothetis NK (2008b) Direct measurement of oxygen extraction with fMRI using 6\% CO2 inhalation. Magn Reson Imaging 26:961-967. 